Recepción: 18 / 05 / 2018

Aceptación: 27 / 07 / 2018

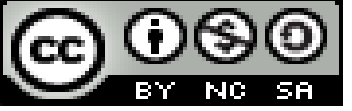

Ciencias técnicas y aplicadas

Publicación: 15 / 09 / 2018

Artículo de investigación

\title{
Control estadístico de procesos en la fabricación de tornillos para aplicaciones domesticas e industriales
}

\section{Statistical control of processes in the manufacture of screws for domestic and industrial application}

\section{Controle estatístico de processos na fabricação de parafusos para aplicações domésticas e industriais}

\author{
Eder L. Cruz-Siguenza ${ }^{\mathrm{I}}$ \\ eder.cruz@espoch.edu.ec \\ Wilson J. Villagrán-Cáceres II \\ wvillagran@espoch.edu.ec \\ Edgar F. Sánchez-Carrión III \\ esanchez_c@espoch.edu.ec, \\ Edwin R. Pozo-Safla IV \\ edwin.pozo@espoch.edu.ec \\ Christian G. Arévalo-Flores V \\ giovanni.flores@espoch.edu.ec
}

Correspondencia: eder.cruz@espoch.edu.ec

\footnotetext{
I Magister en Gestión de la Calidad y Productividad, Ingeniero Industrial Mención Gestión de Procesos, Docente de la Escuela Superior Politécnica de Chimborazo, Riobamba, Ecuador.

II Magister en Matemática Básica, Ingeniero en Electrónica y Computación, Tecnólogo en Informática Aplicada, Docente de la Escuela Superior Politécnica de Chimborazo, Riobamba, Ecuador.

III Magister en Diseño Producción y Automatización Industrial, Ingeniero Mecánico, Docente de la Escuela Superior Politécnica de Chimborazo, Riobamba, Ecuador.

IV Magister en Diseño Producción y Automatización Industrial, Ingeniero Mecánico, Docente de la Escuela Superior Politécnica de Chimborazo, Riobamba, Ecuador.

${ }^{v}$ Estudiante de Ingeniería Mecánica de la Escuela Superior Politécnica de Chimborazo, Riobamba, Ecuador.
} 


\section{Resumen}

En este artículo se analiza el control de calidad de un proceso mediante el cual una empresa busca satisfacer las necesidades del cliente, nos centramos en el control de calidad de los tornillos, debido a que estos son elementos que no se les toma con mucha importancia, pero debido a la necesidad de estos es que algunos objetos cumplen su función, como una cama, un mueble e incluso en máquinas y sistemas, como bombas y motores, sin los tornillos ninguno de estos podría cumplir su función. Entonces para analizar la calidad de máquinas grandes primero debemos hacerlo parte por parte empezando por sus elementos más pequeños y después que ya todo este ensamblado lo hacemos como un todo. Obtuvimos los datos de 25 muestras cada una con 50 tornillos, donde realizamos una gráfica NP para analizar la variación de los artículos defectuosos, donde llegamos a la conclusión de que deben realizarse cambios en el proceso de producción para llegar a la calidad necesaria y satisfacer las necesidades del cliente. También analizamos las posibles causas de porque los clientes no están conformes con ciertos artículos, para esto realizamos un diagrama de Pareto y los problemas con los porcentajes más altos fueron: Baja resistencia a la torsión, baja resistencia a la tracción y baja dureza en el núcleo, donde deberíamos realizar más pruebas para evitar estos problemas.

\section{Palabras claves:}

\section{Abstract}

In this article we analyze the quality control of a process by which a company seeks to satisfy the needs of the client, we focus on the quality control of the screws, because these are elements that are not taken very seriously, but due to the need of these is that some objects fulfill their function, such as a bed, a piece of furniture and even in machines and systems, such as pumps and motors, without the screws none of these could fulfill their function. So to analyze the quality of large machines we must first do part by part starting with its smaller elements and after all this assembly we do as a whole. We obtained the data of 25 samples each with 50 screws, where we made an NP graph to analyze the variation of the defective items, where we reached the conclusion that changes must be made in the production process to reach the necessary quality and satisfy the customer needs. We also analyze the possible causes of why the clients are not satisfied with certain articles, for this we made a Pareto diagram and the problems with the 
highest percentages were: Low torsional strength, low tensile strength and low hardness in the nucleus, where we should do more tests to avoid these problems.

\section{Keywords:}

\section{Resumo}

Neste artigo analisamos o controle de qualidade de um processo pelo qual uma empresa busca satisfazer as necessidades do cliente, nos concentramos no controle de qualidade dos parafusos, pois estes são elementos que não são levados muito a sério, mas devido à necessidade destes, alguns objetos cumprem sua função, como uma cama, uma peça de mobília e até máquinas e sistemas, como bombas e motores, sem os parafusos nenhum deles poderia cumprir sua função. Assim, para analisar a qualidade das máquinas de grande porte, devemos primeiro fazer parte por peça, começando com seus elementos menores e, depois de toda essa montagem, fazemos como um todo. Obtivemos os dados de 25 amostras, cada uma com 50 parafusos, onde fizemos um gráfico NP para analisar a variação dos itens defeituosos, onde chegamos à conclusão de que mudanças devem ser feitas no processo de produção para alcançar a qualidade necessária e satisfazer a demanda. Necessidades do cliente. Analisamos também as possíveis causas de porque os clientes não estão satisfeitos com determinados artigos, para isso fizemos um diagrama de Pareto e os problemas com os maiores percentuais foram: Baixa resistência à torção, baixa resistência à tração e baixa dureza no núcleo, onde devemos fazer mais testes para evitar esses problemas.

\section{Palavras chave:}

\section{Introducción}

El control de calidad es un proceso mediante el cual una empresa busca garantizar que la calidad del producto se mantenga o mejore con errores reducidos o cero. El control de calidad requiere que la empresa cree un entorno en el que tanto la gerencia como los empleados se esfuercen por la perfección. Esto se lleva a cabo capacitando al personal, creando puntos de referencia para la calidad del producto y probando productos para verificar variaciones estadísticamente significativas. 


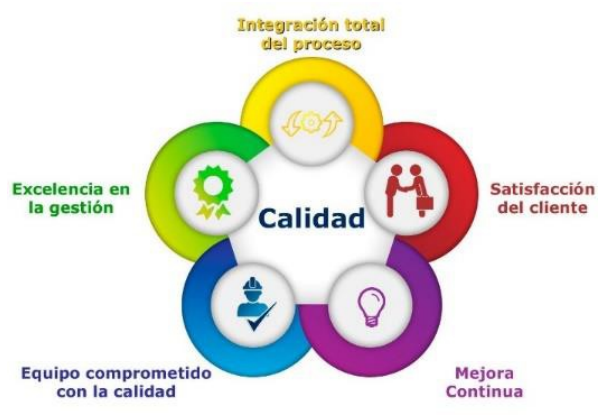

Figura1. Control de Calidad

Un tornillo es una de las seis máquinas simples, y las otras cinco son la palanca, la rueda y el eje, la polea, el plano inclinado y la cuña. Es uno de los dispositivos más comúnmente utilizados en el mundo. El tornillo tiene la apariencia de una delgada barra de metal con una cabeza giratoria en la parte superior y ranuras a lo largo de su longitud. Convencionalmente, se define como un dispositivo mecánico que tiene planos inclinados en hélice y es capaz de convertir el par en fuerza lineal y viceversa.

Los tornillos son muy importantes hoy en día en la industria, ya que son partes de máquinas grandes, bombas, etc., y si llegan a fallar o romperse, esto provoca que todo un sistema deje de funcionar y grandes pérdidas para las empresas, por lo que es importante un riguroso control de calidad en los tornillos. El tipo de rosca, el diseño del punto y el tipo de cabeza de un tornillo determinan las aplicaciones y los tipos de materiales que se deben sujetar. Es por eso que necesita hacerlo bien, por lo que su trabajo es de calidad. Los tornillos para madera tienen un tipo de hilo y punta diferentes a los desarrollados para los metales, por lo que se sujetan mejor y duran más tiempo en ambientes propensos a la putrefacción. Entonces la calidad de los tornillos se puede medir de acuerdo a su aplicación, no es lo mismo un tornillo para armar un mueble que no está sometido a fuerzas tan grandes, como lo es un tornillo que es parte de una bomba, donde la fuerza que va a soportar es muy alta.

Los tornillos precisamente llegan a la industria para mejorar la calidad de ciertos productos ya que anteriormente se utilizaban clavos. Pero los clavos tienen la tendencia a doblarse cuando se usan, mientras que un tornillo nunca se dobla ya que la fuerza se extiende uniformemente a lo largo de todo el eje, los planos inclinados que forman los hilos se enrollan completamente a lo 
largo del eje del tornillo y, por lo tanto, es más fácil fijar diferentes objetos, ya que hacerlo descender en forma giratoria requiere menos esfuerzo que empujar un clavo mediante la aplicación de esfuerzo en una dirección vertical. Estas son algunas razones por las cuales se prefiere usar un tornillo sobre un clavo, y por qué este último se está eliminando lentamente.

\section{Fabricación de Tornillos}

Para la fabricación de tornillos primero hay que cumplir ciertos parámetros y normas de calidad que han venido cambiando con el tiempo de la siguiente manera:

En 1928, la Comisión Nacional de Roscas de Tornillo fue creada para ese propósito. En 1948 se adoptó un sistema de rosca de tornillo unificado que se enfocaba en la cantidad de hilos por pulgada, la imagen y los tamaños de rosca y diámetro. En 1966, la Organización Internacional de Estándares ayudó a crear restricciones universales en los rangos de tamaño métrico y en pulgadas. Estos estándares fueron finalmente aceptados como el estándar global.

Cuando se crea por primera vez un tornillo, los fabricantes primero comienzan con un proceso llamado encabezado en frío. El alambre se alimenta desde una bobina mecánica a través de una máquina de pre enderezado. El cable fluye directamente a una máquina que corta automáticamente el cable en su punto designado y también corta la cabeza del tornillo en bruto en su forma deseada. La máquina de encabezado utiliza un dado abierto o cerrado que requiere uno o dos golpes para crear la cabeza del tornillo. El dado cerrado crea un blanco de tornillo preciso. En promedio, la máquina de encabezado en frío produce 100-500 troqueles de tornillo por minuto. Una vez que el tornillo está en frío, los espacios en blanco se alimentan automáticamente a los troqueles de corte de rosca de una tolva vibrante. La tolva guía los espacios en blanco de los tornillos por un canal inclinado hacia los moldes mientras se asegura que el tornillo esté en la posición correcta para ser alimentado a la máquina.

El blanco se corta utilizando una de las tres técnicas de fabricación de tornillos. En la matriz alternativa, se utilizan dos troqueles planos para cortar la rosca. Un dado permanece quieto, mientras que el otro se mueve de manera alternativa. El espacio en blanco se rueda entre los dos. 
Cuando se utiliza la matriz cilíndrica, la pieza en bruto del tornillo se enrolla entre los dos o tres troqueles redondos para terminar la rosca. La etapa final del laminado del hilo es el proceso de troquelado rotatorio planetario. Este proceso mantiene el tornillo en su lugar mientras que varias máquinas de corte ruedan alrededor del blanco para crear el producto terminado. Estos métodos crean tornillos de mayor calidad y conservan una mayor durabilidad. Esto se debe a que el hilo no está cortado, sino impresionado en el espacio en blanco. No se pierde material y se evita la debilidad en el metal. Los hilos se posicionan con precisión y las técnicas de laminado de roscas permiten que se repitan más tornillos, hasta 2000, partes por minuto.

Los materiales más comunes en los que se fabrican los tornillos hoy en día están compuestos de alambre de acero con poco carbono medio. Otros materiales utilizados incluyen latón, acero inoxidable, aleación de níquel o aluminio. El acero se puede platear con zinc, níquel, cromo u otros materiales de recubrimiento. Otros tornillos tienen materiales de acabado adicionales para protección adicional y deben ser compatibles con las materias primas de las que está hecho el tornillo.

\section{Tipos de Tornillos}

Hay muchas variedades diferentes de tornillos que se seleccionan según los requisitos particulares o los materiales involucrados. Algunos de los tipos más comunes incluyen:

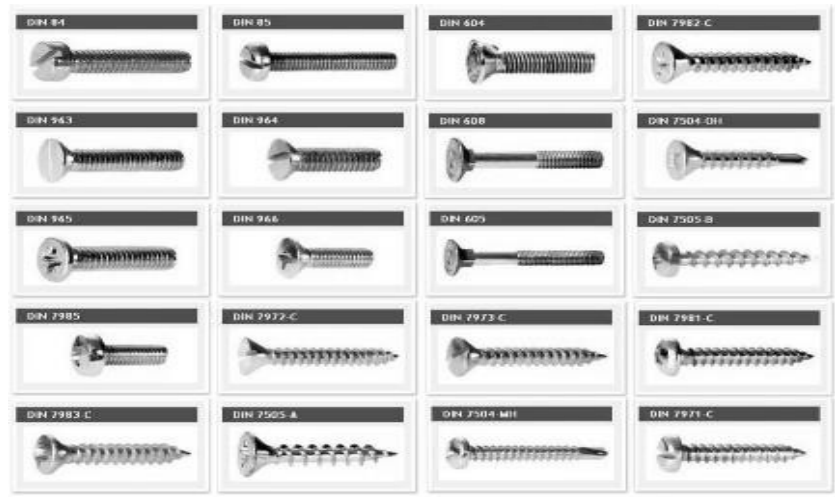

Figura 2. Tipos de Tornillos

\section{Clasificación de tornillos según el requisito de trabajo}




\section{Tornillo de madera}

Estos tornillos se usan en madera y vienen en varios tipos y tamaños de cabezales. Hecho de hilos gruesos para obtener un buen agarre al penetrar la madera.

\section{Tornillo de chapa}

Estos tornillos también vienen en varios tipos de cabezal y tamaños e hilos completos a lo largo de la longitud del tornillo auxiliar en aplicaciones relacionadas con chapas metálicas. También se usa en aplicaciones que requieren la unión de diferentes materiales.

\section{Tornillo penetrante}

Puede ser en forma de tornillos de chapa, madera o paneles de yeso. Estos tornillos se perforan en el material, creando sus propios agujeros. Elimina la necesidad de perforar previamente.

\section{Tornillo Lag}

Uno de los sujetadores más difíciles en uso hoy en día. Se utiliza para unir materiales pesados, como madera. Son diferentes de la madera genérica y chapa.

\section{Tornillo de MDF}

Los tornillos de tablero de fibra de densidad media ayudan en aplicaciones como el montaje de estanterías. Por lo general, son más duros que la madera sólida y, por lo tanto, son más difíciles de perforar. Requiere perforación previa.

\section{El tornillo de la máquina}

Se utiliza para sujetar y guardar materiales con orificios pre taladrados en su lugar. También se usa en conexiones eléctricas, regletas de bornes y carcasas de motores.

\section{Tornillo de aglomerado}

Se usa para mantener juntos el tablero de partículas de diferentes densidades, como bajo, medio y alto. Completamente roscado ayuda de la espiga en su aplicación.

\section{Tornillo de aglomerado}

A menudo se recubre con cera y se usa para sujetar pisos de madera aglomerada.

\section{Tornillo de espejo}

Diseñado con una cúpula decorativa u otra cubierta para ocultar la cabeza. 


\section{Tornillo de cabeza de seguridad}

Diseñado con una cabeza que es imposible de invertir, por lo que es adecuado para aplicaciones de seguridad.

\section{Tornillo de plataforma}

Compuesto de hilos gruesos y una caña lisa como tornillos de madera. Hecho para resistir el óxido y la corrosión. Ayuda en la cubierta de fijación y se utiliza con cubiertas de madera tratadas a presión.

\section{Tornillo de accionamiento}

Suave, redondo o con cabeza de hongo con mango de diámetro reducido.

\section{Tornillo de yeso}

A menudo cubierto con fosfato negro y diseñado con una cabeza de corneta. Se utiliza para unir paneles de yeso a vigas de madera o metal.

\section{Cáncamo}

Una cabeza en bucle diseñada para ser utilizada como un punto de fijación. También se usa para unir cables en las superficies de construcción.

\section{Tornillo de ajuste}

Tornillos sin cabeza que se utilizan para entrar por completo en los orificios roscados. Puede sostener un objeto a otro objeto sin el uso de una tuerca.

\section{Clasificación de pernos:}

\section{Perno de carro}

Utilizado para unir metal a la madera. Recorte cuadrado de la cabeza del perno para asegurar que el perno esté en su lugar cuando se lo fija en su lugar.

\section{Perno de cabeza hexagonal}

Consiste en seis cabezas laterales. Comúnmente utilizado para aplicaciones en madera o metal. 


\section{Perno de hombro}

Se usa en poleas, engranajes y otras partes móviles ya que permite el movimiento de los componentes vinculados.

\section{Perno estructural}

Típicamente tienen una longitud de hilo corta que los pernos hexagonales. Utilizado en aplicaciones estructurales.

\section{Perno de cabeza cuadrada}

Uso en la industria, agrícola y de la construcción. La forma cuadrada facilita el uso en áreas estrechas.

\section{Perno J}

Úselo en conexiones estructurales para sujetar paredes a cimientos de hormigón.

\section{Perno U}

Usar en tuberías a través de las cuales fluyen líquidos como líquidos y gases.

\section{Perno del elevador}

Utilizado en elevadores y sistemas de cintas transportadoras. El gran diámetro de la cabeza evita que el perno penetre en el material del transportador.

\section{Perno de retraso}

También conocido como tirafondos. No requiere orificios pre taladrados, ya que produce hilo propio en la madera durante la penetración.

\section{Perno de brida}

Consiste en una arandela en la parte inferior de la cabeza del perno. Ayuda en la distribución de la carga en el perno.

\section{Perno de anclaje}

Usado en aplicaciones estructurales para sujetar materiales al concreto o mampostería. 


\section{Perno de arado}

Pernos muy duraderos debido a la cabeza plana seguida de un mango cuadrado. Uso en construcción.

\section{Perno de madera}

Usar en tablones y estructuras de madera grandes. Comúnmente utilizado en industrias de marina y madera.

\section{Partes de un Tornillo}

Ranura: es donde va la punta de su destornillador. También se conoce como cabeza de tornillo.

Cabeza: la cabeza es la parte superior del tornillo. Es típicamente más ancho que el mango y el hilo. Las cabezas pueden ser planas o abovedadas. Algunos tienen un diámetro pequeño en relación con el mango y otros son muy anchos.

Longitud del hilo: la longitud del hilo es de longitud completa o parcial. En consecuencia, hay tornillos de rosca completa y rosca parcial. Los tornillos de rosca parcial son más fuertes en el sentido de que la parte que no es de hilo es más fuerte sin puntos débiles. Cuando necesite atornillar algo junto con la fuerza máxima, lo mejor es un tornillo o perno de rosca parcial.

Punto: El punto es, obviamente, donde el tornillo ingresa el material en el que está colocando el tornillo. Si no tiene punto (es decir, punta plana), es un perno que requiere un agujero prefabricado.

El hilo: Sorprendentemente, el hilo tiene muchos componentes y varía entre los tornillos. Está el ángulo del hilo, que es lo abruptamente que se inclina hacia abajo. También hay distancia entre los hilos que hacen la diferencia. Algunos tornillos tienen hilos pequeños y muy juntos. Otros tornillos tienen hilos más grandes espaciados más separados (es decir, anclajes de paneles de yeso). 
Grosor del tornillo (diámetro menor y mayor): hay dos medidas cuando se trata del grosor del tornillo. Hay diámetros menores y mayores. El diámetro principal es el diámetro en la parte más gruesa del hilo (es decir, la parte exterior del hilo). El diámetro menor es el grosor en la base del tornillo.

Cresta: Cresta es la punta y el valle del hilo.

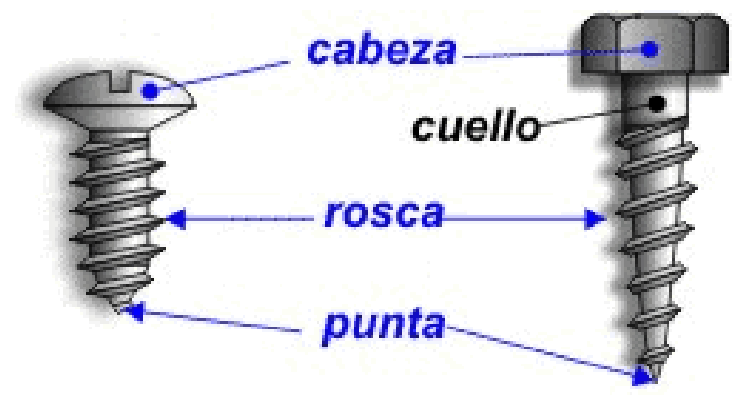

Figura 3. Partes de un tornillo

\section{Cálculos del Control de Calidad de Tornillos}

Se desea construir un gráfico de control, para controlar un proceso que fabrica tornillos que se aplican para fijar bombas. Se tienen 25 muestras, cada una formada por 50 tornillos. El número de tornillos defectuosos en cada una de las muestras se muestra en la tabla siguiente:

Tabla 1. Defectos en tornillos

\begin{tabular}{|c|c|c|c|r|r|r|}
\hline Muestra & $\begin{array}{l}\text { Número de } \\
\text { Articulos }\end{array}$ & $\begin{array}{l}\text { Articulos } \\
\text { Defectuosos }\end{array}$ & $\begin{array}{c}\text { PROPORCI } \\
\text { ON Pi }\end{array}$ & LCI & LC & LCS \\
\hline 1 & 50 & 3 & 0.06 & -1.87 & 3.64 & 9.15 \\
\hline 2 & 50 & 5 & 0.1 & -1.87 & 3.64 & 9.15 \\
\hline 3 & 50 & 5 & 0.1 & -1.87 & 3.64 & 9.15 \\
\hline 4 & 50 & 1 & 0.02 & -1.87 & 3.64 & 9.15 \\
\hline 5 & 50 & 10 & 0.2 & -1.87 & 3.64 & 9.15 \\
\hline 6 & 50 & 4 & 0.08 & -1.87 & 3.64 & 9.15 \\
\hline 7 & 50 & 2 & 0.04 & -1.87 & 3.64 & 9.15 \\
\hline 8 & 50 & 5 & 0.1 & -1.87 & 3.64 & 9.15 \\
\hline 9 & 50 & 6 & 0.12 & -1.87 & 3.64 & 9.15 \\
\hline 10 & 50 & 4 & 0.08 & -1.87 & 3.64 & 9.15 \\
\hline 11 & 50 & 1 & 0.02 & -1.87 & 3.64 & 9.15 \\
\hline 12 & 50 & 0 & 0 & -1.87 & 3.64 & 9.15 \\
\hline 13 & 50 & 4 & 0.08 & -1.87 & 3.64 & 9.15 \\
\hline 14 & 50 & 6 & 0.12 & -1.87 & 3.64 & 9.15 \\
\hline 15 & 50 & 2 & 0.04 & -1.87 & 3.64 & 9.15 \\
\hline 16 & 50 & 2 & 0.04 & -1.87 & 3.64 & 9.15 \\
\hline 17 & 50 & 3 & 0.06 & -1.87 & 3.64 & 9.15 \\
\hline 18 & 50 & 4 & 0.08 & -1.87 & 3.64 & 9.15 \\
\hline 19 & 50 & 2 & 0.04 & -1.87 & 3.64 & 9.15 \\
\hline 20 & 50 & 5 & 0.1 & -1.87 & 3.64 & 9.15 \\
\hline 21 & 50 & 4 & 0.08 & -1.87 & 3.64 & 9.15 \\
\hline 22 & 50 & 5 & 0.1 & -1.87 & 3.64 & 9.15 \\
\hline 23 & 50 & 2 & 0.04 & -1.87 & 3.64 & 9.15 \\
\hline 24 & 50 & 4 & 0.08 & -1.87 & 3.64 & 9.15 \\
\hline 25 & 50 & 2 & 0.04 & -1.87 & 3.64 & 9.15 \\
\hline & & & & & & \\
\hline
\end{tabular}

$p=\frac{\Sigma P i}{\text { \#muestras }}$

$p=\frac{1.82}{25}$ 


$$
\begin{aligned}
& \boldsymbol{p}=\mathbf{0 . 0 7 2 8} \\
& \boldsymbol{n}=\mathbf{5 0} \\
& n p=0.0728 \times 50 \\
& \boldsymbol{L C}=n \boldsymbol{p}=\mathbf{3 . 6 4} \\
& L C I=n p-3 \sqrt{n p(1-p)} \\
& L C I=3.64-3 \sqrt{3.64(1-0.0728)} \\
& \mathbf{L C I}=-\mathbf{1 . 8 7} \\
& L C S=n p+3 \sqrt{n p(1-p)} \\
& L C S=3.64+3 \sqrt{3.64(1-0.0728)} \\
& \mathbf{L C S}=\mathbf{9 . 1 5}
\end{aligned}
$$

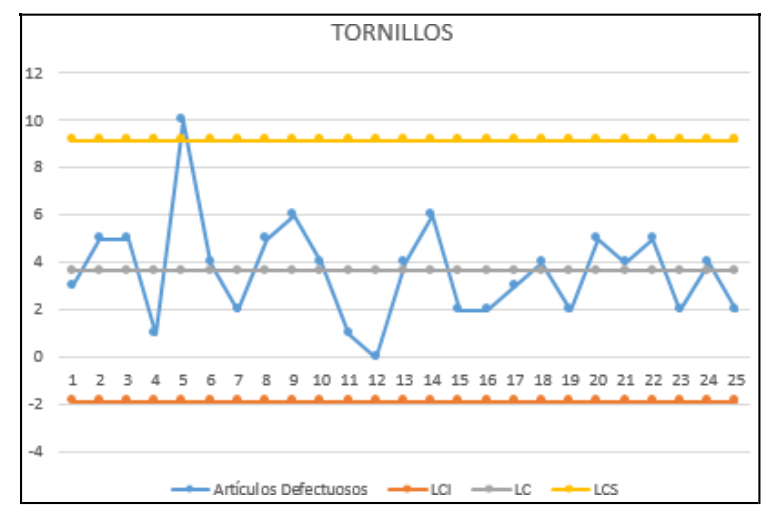

Figura 4. Gráfica NP Excel

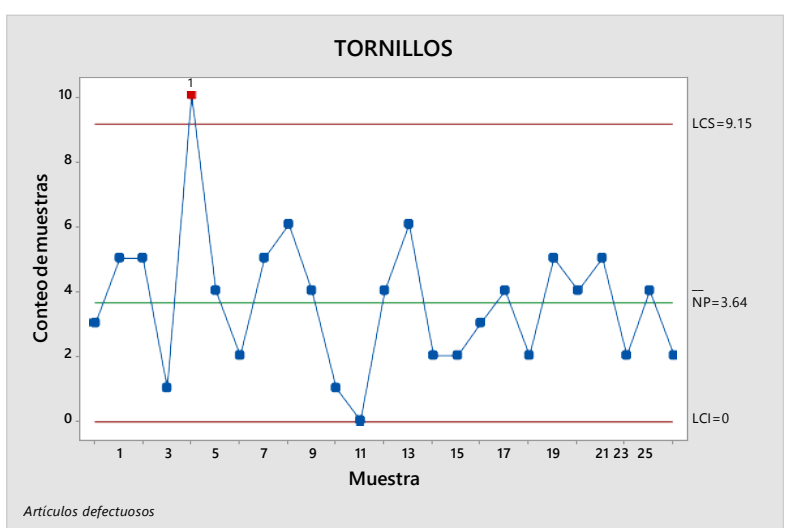

Figura 5. Gráfica NP Minitab

Desviación Estándar $=\sigma=2.099$

$c p=\frac{L C S-L C I}{6 \sigma}$

\section{6}




$$
\begin{aligned}
& c p=\frac{9.15+1.87}{6 \times 2.099} \\
& c p=\mathbf{0 . 8 7 5} \\
& c p k i=\frac{L C S-L C}{3 \sigma} \\
& c p k i=\frac{9.15-3.64}{3 \times 2.099} \\
& c p k i=\mathbf{0 . 8 7 5} \\
& c p k s=\frac{L C-L C I}{3 \sigma} \\
& c p k s=\frac{3.64-1.87}{3 \times 2.099} \\
& c p k s=\mathbf{0 . 8 7 5} \\
& c p k=\mathbf{0 . 8 7 5}
\end{aligned}
$$

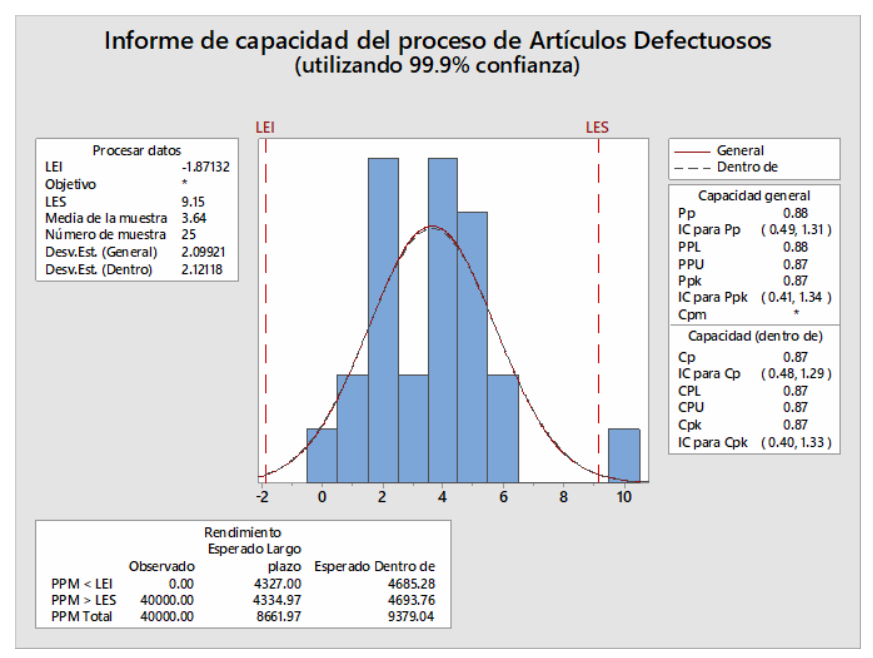

Figura 6. Análisis de $\mathrm{cp}$

Una empresa que fabrica tornillo, ha encontrado que el número de clientes insatisfechos en último mes se les ha disparado, y se plantean, en primer lugar, buscar los motivos que han producido estas desconformidades, para después atajar el problema. Los motivos se encuentran detallados en la siguiente tabla.

Tabla 2. Causas de defectos en tornillos 


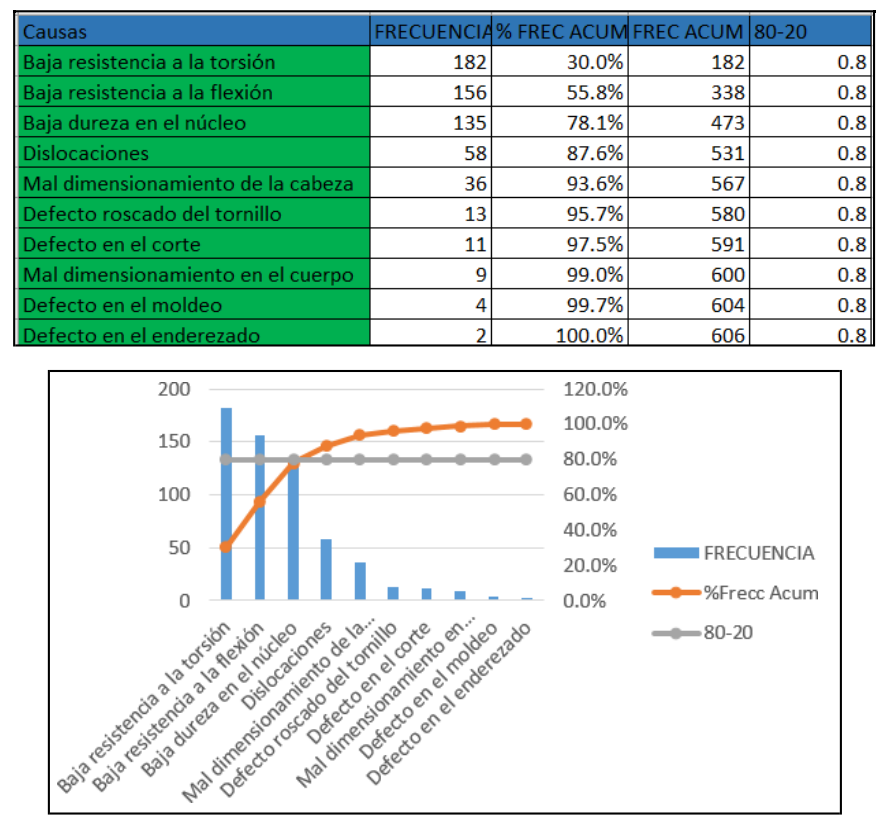

Figura 7. Pareto Excel

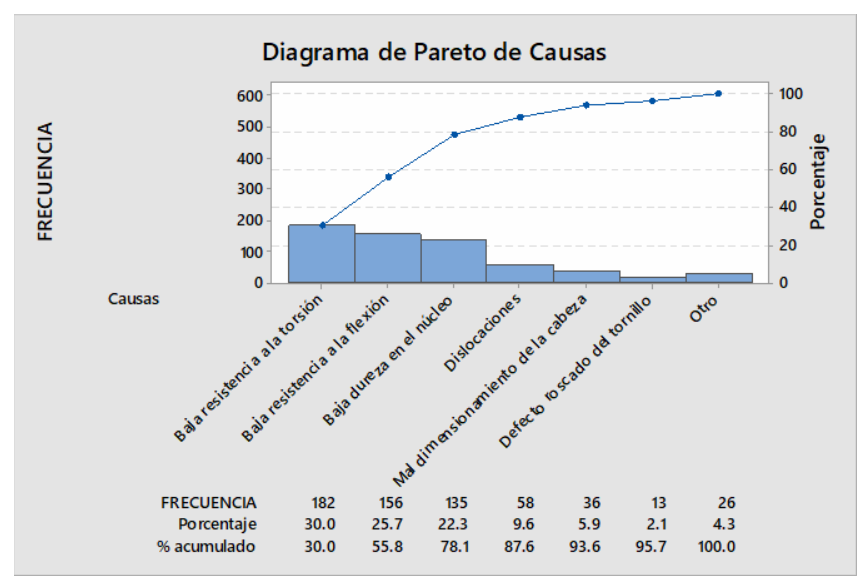

Figura 8. Pareto Minitab

\section{Análisis y Discusión de Resultados}

Para analizar el número de tornillos defectuosos, que se utilizan para fijar bombas. Se utilizó una gráfica NP, esto debido a que todos sus lotes son de 50 unidades. En está gráfica podemos observar que la mayoría de los puntos están dentro de los limites correspondientes, pero hay que poner mucha atención para que el proceso sea más regular ya un punto está fuera del límite y otro se encuentra justo en el límite, entonces hay que poner mucha atención especialmente en esos lotes. Pero a pesar de que los otros puntos se encuentran dentro de los límites hay que tratar de que el proceso sea un poco más estable, ya que la gráfica que observamos está muy irregular, entonces hay que corregir esa irregularidad para que existan menos fallas en los tornillos y la 
calidad de estos sea mejore continuamente. Debido a que el cp y cpk son menores que uno, debemos realizar un análisis de la producción y realizar cambios necesarios para que la calidad llegue hacer satisfactoria y de conformidad al cliente.

Después analizamos la desconformidad de algunos clientes, ya que el producto tenia ciertos defectos. Entonces enlistamos las causas por las cuales los tornillos no daban conformidad al cliente, y con eso procedimos a realizar un diagrama de Pareto, donde nos dio como resultado que, la baja resistencia a la torsión, la baja resistencia a la flexión y la baja dureza del núcleo son los problemas que más comúnmente o en alto porcentaje hacen que el producto falle y por ende la queja de los clientes. Para esto debemos realizar más pruebas en el producto y evitar que dichos problemas sigan afectando a la producción, y sin dejar de lado las causas que no tienen alto porcentaje, pero sin embargo son un problema y también perjudica a la calidad del producto.

\section{Conclusiones}

El tornillo aparenta ser un elemento de poca importancia, pero si este llega a fallar, se ve perjudicada la calidad de máquinas y sistemas importantes como: bombas, motores, bridas. Entonces para controlar la calidad de estos productos se lo hace primero parte por parte, ya que todos los elementos de las máquinas cumplen un papel importante incluido los tornillos.

El momento de escoger un tornillo, debemos saber en qué circunstancias va a trabajar, si va tener que soportar fuerzas grandes o pequeñas, si va a estar sometido a ambientes húmedos o talvez va a estar bajo el agua, solo así podemos decidir las características del tornillo como: forma, tamaño, material. Ahí observamos si falla o no y por ende la calidad del producto.

En toda elaboración de un producto existen fallas, por lo que debemos realizar las pruebas necesarias para identificar el error y así poder corregirlo. Por esta razón existe el control de calidad de los productos con sus normas respectivas.

En muchos de los trabajos que realizamos en la vida cotidiana; uno de los elementos más utilizados es el tornillo, ya que este garantiza la sujeción y la unión de las diferentes piezas en que el usuario trabaja, esta seguridad no proporciona otros elementos como los clavos.

El tornillo es un sujetador que sirve para armar y desarmar objetos grandes y pesados, y así poder trasladarlos con más facilidad. 


\section{Recomendaciones}

El momento de requerir un tornillo, recurrir a los catálogos ya que una mala decisión el momento de escoger, puede provocar daños en máquinas grandes y por ende muchas pérdidas en empresas.

Cuando se remplace un tornillo, tratar de que tenga las mismas características o por lo menos similares. Pero nunca se remplace por uno que tenga menos resistencia que el anterior, porque es casi seguro que va a fallar.

\section{Referencias Bibliográficas}

RAMIREZ WENDY, Pernos y Tornillos, Moquegua 2013. Recuperado de: https://es.scribd.com/doc/141395363/tipos-de-tornillos-y-tuercas-pdf-pdf

MOLINERO LUIS, Control de Calidad, España 2003, Recuperado de: https://www.alceingenieria.net/bioestadistica/calidad.pdf

INGEMECANICA, Estudio y Tipología de las Uniones Atornilladas, 2018. Recuperado de: https://ingemecanica.com/tutorialsemanal/tutorialn31.html

PARTESDEL, Partes del tornillo, 2018. Recuperado de: https://www.partesdel.com/tornillo.html

RÍOS ALEJANDRA, Herramientas básicas para el control de calidad, 2009, Recuperado de: https://es.slideshare.net/alerioz/7-herramientas-bsicas-para-el-control-de-la-calidad. 